\title{
EMBEDDING OF A LIE ALGEBRA INTO LIE-ADMISSIBLE ALGEBRAS
}

\author{
HYO CHUL MYUNG
}

\begin{abstract}
Let $A$ be a flexible Lie-admissible algebra over a field of characteristic $\neq 2,3$. Let $S$ be a finite-dimensional classical Lie subalgebra of $A^{-}$which is complemented by an ideal $R$ of $A^{-}$. It is shown that $S$ is a Lie algebra under the multiplication in $A$ and is an ideal of $A$ if and only if $S$ contains a classical Cartan subalgebra $H$ which is nil in $A$ and such that $H H \subseteq S$ and $[H, R]=0$. In this case, the multiplication between $S$ and $R$ is determined by linear functionals on $R$ which vanish on $[R, R]$. If $A$ is finite-dimensional and of characteristic 0 then this can be applied to give a condition that a Levi-factor $S$ of $A^{-}$be embedded as an ideal into $A$ and to determine the multiplication between $S$ and the solvable radical of $A^{-}$.
\end{abstract}

1. Introduction. For an algebra $A$, denote by $A^{-}$the algebra with multiplication $[x, y]=x y-y x$ defined on the vector space $A$. If $A^{-}$is a Lie algebra then $A$ is said to be Lie-admissible. If $A$ satisfies the flexible law $(x y) x=x(y x)$ for all $x, y \in A$ then $A$ is called a flexible algebra. A linearized form of the flexible law is $(x y) z-x(y z)+(z y) x-z(y x)=0$. If $A$ is flexible and Lie-admissible then it is well known that the mapping ad $x$ : $a \rightarrow[a, x]$ is a derivation of $A$ for all $x \in A$; that is, $[a, b c]=[a, b] c+$ $b[a, c]$ for all $a, b, c \in A$. Recent results show that a Cartan subalgebra of $A^{-}$plays a major role for the structure of flexible Lie-admissible algebras $A$ [1], [2], [3]. Possible applications of Lie-admissible algebras in physics have been recently pointed out by a number of physicists. For this, the reader is referred to Santilli's recent work [5].

The purpose of this paper is to give a condition in terms of a Cartan subalgebra that a classical Lie algebra $S$ be embedded as an ideal into a flexible Lie-admissible algebra $A$ when the subspace $S$ is complemented by an ideal $R$ of $A^{-}$, and then to determine the multiplication between $S$ and $R$. We make use of the known structure for classical Lie algebras [4] and the result that if $A$ is a flexible algebra and $A^{-}$is a classical Lie algebra with a classical Cartan subalgebra which is nil in $A$, then $A$ is a Lie algebra isomorphic to $A^{-}$[2]. A subset $M$ of $A$ said to be nil in $A$ if every element of $M$ is power-associative and nilpotent in $A$.

Received by the editors March 27, 1978.

AMS (MOS) subject classifications (1970). Primary 17A20, 17B05.

Key words and phrases. Flexible algebra, Lie-admissible algebra, classical Lie algebra, Cartan subalgebra, Levi-factor. 
Let $S$ be the direct sum of Lie algebras $S_{1}, \ldots, S_{n}$ over a field $\Phi$ of characteristic $\neq 2$ and $R$ be a flexible Lie-admissible algebra over $\Phi$. Let $f_{1}, \ldots, f_{n}$ be linear functionals on $R$ which vanish on $[R, R]$. Define a multiplication in the vector space direct sum $A=S+R$ as follows:

$$
\begin{aligned}
\left(\sum_{i=1}^{n} x_{i}+r\right)\left(\sum_{i=1}^{n} y_{i}+s\right)=\sum_{i=1}^{n}\left[x_{i} y_{i}+f_{i}(r) y_{i}+f_{i}(s) x_{i}\right]+r s, & \\
x_{i}, y_{i} \in S_{i}, r, s \in R, i & =1,2, \ldots, n .
\end{aligned}
$$

Then one sees that $[x+r, y+s]=2 x y+[r, s]$ for $x, y \in S$ and $r, s \in R$ and so $A$ is Lie-admissible. One also computes

$$
[(x+r)(y+s)](x+r)-(x+r)[(y+s)(x+r)]=\sum_{i} f_{i}([r, s]) x_{i}=0
$$

where $x=\Sigma_{i} x_{i}$, since $f_{i}([R, R])=0$. Thus $A$ is flexible. Clearly, $R$ is a subalgebra of $A$ and is an ideal of $A^{-}$. The Lie algebra $S$ is embedded as an ideal in $A$ and if $x \in S_{i}$ then $x r=r x=f_{i}(r) x$ for $r \in R$. In fact, we show that this is the essential source for the embedding in question. We state the main result as follows.

THEOREM 1. Let $A$ be a flexible Lie-admissible algebra over a field $\Phi$ of characteristic $\neq 2,3$ (not necessarily finite-dimensional). Let $S$ be a finite-dimensional classical subalgebra of $A^{-}$which is complemented by an ideal $R$ of $A^{-}$. Then $S$ is a Lie algebra under the multiplication in $A$ and is an ideal of $A$ if and only if $S$ contains a classical Cartan subalgebra $H$ which is nil in $A$ and such that $H H \subseteq S$ and $[H, R]=0$. In this case, $R$ is a subalgebra of $A$ and the multiplication in $A$ is given by the rule (*) where $f_{1}, \ldots, f_{n}$ are linear functionals on $R$ which vanish on $[R, R]$, and $n$ is the number of simple summands in $S$.

Recall that a finite-dimensional classical Lie algebra is a direct sum of simple Lie algebras [4].

2. Proof of Theorem 1. We begin with the following lemma.

LEMMA. Let $L$ be a Lie algebra over an arbitrary field $\Phi$. Let $S$ be a finite-dimensional subalgebra of $L$ and $H$ be a Cartan subalgebra of $S$. Then, for an ideal $R$ of $L,[S R]=0$ if and only if $[H R]=0$.

Proof. One may assume that $\Phi$ is algebraically closed; if not, one takes the scalar extension of $S$ to the algebraic closure of $\Phi$. Let $S=\Sigma_{\alpha} S_{\alpha}$ be the Cartan decomposition for $S$ relative to $H$. For each nonzero root $\alpha$, choose an $h \in H$ such that $\alpha(h) \neq 0$. Then ad $h: S_{\alpha} \rightarrow S_{\alpha}$ is surjective; for, if not then there is an element $x \neq 0$ in $S_{\alpha}$ such that $[x, h]=0$ and this together with $x(\operatorname{ad} h-\alpha(h) I)^{n}=0$ implies that $\alpha(h)^{n} x=0$. This is absurd. Thus we have that $\left[S_{\alpha} h\right]=S_{\alpha}$ for $\alpha \neq 0$ and $\alpha(h) \neq 0$. If $[H R]=0$ and $\alpha \neq 0$ then, by the Jacobi identity, $\left[S_{\alpha} R\right]=\left[\left[S_{\alpha} h\right] R\right] \subseteq\left[[R h] S_{\alpha}\right]+\left[\left[S_{\alpha} R\right] h\right]=0$ since $R$ is an ideal of $L$, and so $[S R]=0$.

For the proof of Theorem 1, we first observe that the centralizer $C_{A^{-}}(M)$ of 
a subset $M$ of $A$ in $A^{-}$is a subalgebra of $A$, since $[x y, M] \subseteq x[y, M]+$ $[x, M] y=0$ for all $x, y \in C(M)$. Suppose that $S$ is a finite-dimensional classical subalgebra of $A^{-}$having a classical Cartan subalgebra $H$ which is nil in $A$ and that $H H \subseteq S$ and $[H, R]=0$. That $H H \subseteq S$ implies that $S$ is a subalgebra of $A$ [3]. It then follows from [2, Corollary 3.4] that $S$ is a Lie algebra under the multiplication in $A$. Thus we have that $[x, y]=x y-y x=$ $2 x y$ for $x, y \in S$. By the Lemma, we get $[S, R]=0$ and since $S$ has center 0 , $R=C_{A^{-}}(S)$ and so $R$ is a subalgebra of $A$.

Let $x, y \in S$ and let $r \in R$. Write $y r=z+s$ for some $z \in S$ and $s \in R$. Then the flexible law $(x y) r-x(y r)+(r y) x-r(y x)=0$ implies $(x y) r=x z$ and so $(S S) R \subseteq S$. Since $S S=S$, this proves that $S$ is an ideal of $A$. Since $C_{S}(H)=H$ and $H H=[H, H]=0$, from $\left[h^{\prime}, h r\right]=\left[h^{\prime}, h\right] r+h\left[h^{\prime}, r\right]=0$ for all $h, h^{\prime} \in H$ and $r \in R$, we have

$$
H R=R H \subseteq H .
$$

Let $S=\Sigma_{\alpha} S_{\alpha}$ be the Cartan decomposition for $S$ relative to $H$. Note that $S_{0}=H$ and $\operatorname{dim} S_{\alpha}=1$ for $\alpha \neq 0$ since $S$ is classical. Then $x h=\alpha(h) x$ for $x \in S_{\alpha}$ and $h \in H$. For each nonzero root $\alpha$, choose an $h \in H$ with $\alpha(h) \neq$ 0 . If $x \in S_{\alpha}$ and $r \in R$ then, from $(x h) r-x(h r)+(r h) x-r(h x)=0$ and (1), one gets $x r=\alpha(h)^{-1} \alpha(h r) x$ and so

$$
x r=r x=\lambda x, x \in S_{\alpha}, \quad \alpha \neq 0,
$$

where $\lambda \in \Phi$ depends on $r \in R$ and $\alpha \neq 0$. Since $S_{\alpha} S_{-\alpha}$ is one-dimensional for $\alpha \neq 0$, one chooses nonzero elements $x \in S_{\alpha}, y \in S_{-\alpha}, h \in H$ such that

$$
x h=x, \quad y h=-y, \quad x y=h .
$$

Then by (2) we have that $x r=r x=\lambda x$ and $y r=r y=\mu y$ for $\mu \in \Phi$ and $r \in R$. If $H=\Phi x+B$ is a vector space direct sum then by (1) we can let $h r=r h=\nu h+b, \nu \in \Phi, b \in B$. From (3) and $(x y) r-x(y r)+(r y) x-$ $r(y x)=0$, one gets $2(\nu h+b)=2 \mu h$, so $b=0$ and $\nu=\mu$, and by symmetry $\nu=\lambda$. Therefore

$$
x r=r x=f_{\alpha}(r) x, \quad y r=r y=f_{\alpha}(r) y, \quad h r=r h=f_{\alpha}(r) h
$$

for $\alpha \neq 0$ and $r \in R$, where $f_{\alpha}$ is a linear functional on $R$ and $\{x, y, h\}$ is the canonical basis as in (3). In particular, we have that $f_{\alpha}=f_{-\alpha}$.

Recall that $S$ is the direct sum of simple classical Lie algebras, so that each simple summand has a fundamental system of roots relative to a classical Cartan subalgebra which is connected. Thus we may assume that $S$ is simple. If $\alpha, \beta(\beta \neq 0)$ is an ordered pair of roots then recall the Cartan integer $A_{\alpha, \beta}=r-q$ where $r$ and $q$ are the least nonnegative integers such that $\alpha-(r+1) \beta$ and $\alpha+(q+1) \beta$ are not roots. Let $\Pi=\left\{\alpha_{1}, \ldots, \alpha_{m}\right\}$ be a fundamental system of roots which is connected; that is, for any two roots $\alpha$, $\beta \in \Pi$, there are roots $\mu_{1}, \ldots, \mu_{r} \in \Pi$ such that $\alpha=\mu_{1}, \beta=\mu_{r}$ and $A_{\mu, \mu_{+1}}$ $\neq 0,1<i<r$. For brevity, denote $A_{\alpha_{i} \alpha_{j}}=A_{i j}$ and $f_{\alpha_{i}}=f_{i}$. We first show that if $A_{i j} \neq 0$ then $f_{i}=f_{j}$. If $A_{i j}<0$ then $S_{\alpha_{i}} S_{\alpha_{j}} \neq 0$ and so choose elements 
$x \in S_{\alpha_{i}}, y \in S_{\alpha_{j}}$ with $x y \neq 0$. Then the flexible law $(x y) r-x(y r)+x(y r)-$ $r(y x)=0$ with (4) implies that $(x y) r=f_{j}(r)(x y)$ for all $r \in R$. By symmetry we have that $(x y) r=f_{i}(r)(x y)$ and so $f_{i}=f_{j}$. If $A_{i j}>0$ then, using the relation $A_{\alpha,-\beta}=-A_{\alpha, \beta}$ and $f_{\alpha}=f_{-\alpha}$, we argue that $f_{i}=f_{j}$. Since $\Pi$ is connected, this proves that $f_{i}=f_{j}$ for all $i, j=1,2, \ldots, m$. Denote one of these linear functionals $f_{i}$ by $f$.

Let $x_{i}, y_{i}, h_{i}$ be the canonical basis corresponding to the root $\alpha_{i} \in \Pi$. We have then shown

$$
\begin{aligned}
x_{i} r=r x_{i}=f(r) x_{i}, \quad y_{i} r=r y_{i}=f(r) y_{i}, \quad h_{i} r= & r h_{i}=f(r) h_{i}, \\
& \quad r \in R, i=1,2, \ldots, m .
\end{aligned}
$$

Since, for any root $\alpha \neq 0, \alpha$ or $-\alpha$ is a sum of roots in $\Pi$, there is a basis for $S$ which consists of elements of the form

$$
\begin{aligned}
h_{i},\left(\cdots\left(x_{i_{1}} x_{i_{2}}\right) \cdots x_{i_{k}}\right), \quad & \left(\cdots\left(y_{i_{1}} y_{i_{2}}\right) \cdots y_{i_{k}}\right), \\
& i=1,2, \ldots, m,\left\{i_{1}, \ldots, i_{k}\right\} \subseteq\{1,2, \ldots, m\} .
\end{aligned}
$$

The flexible law $\left(x_{i} x_{j}\right) r-x_{i}\left(x_{j} r\right)+\left(r x_{j}\right) x_{i}-r\left(x_{j} x_{i}\right)=0$ gives $\left(x_{i} x_{j}\right) r=$ $f(r)\left(x_{i} x_{j}\right)$ by (5). Therefore, by induction, we have

$$
x r=r x=f(r) x, \quad x \in S, r \in R .
$$

Finally, let $r, s \in R$ and let $x$ be a nonzero element of $S$. Then $(r s) x-$ $r(s x)+(x s) r-x(s r)=0$ gives $f([r, s])=0$ and so $f$ vanishes on $[R, R]$. Thus the multiplication in $A$ is given by (*) and this completes the proof.

3. Application. Let $A$ be a finite-dimensional flexible Lie-admissible algebra over a field of characteristic 0 . Then, by Levi's theorem, every Levi-factor $S$ (a maximal semisimple subalgebra of $A^{-}$) of $A^{-}$is complemented by the solvable radical of $A^{-}$. It then follows from Corollary of [3] that if $S$ is power-associative in $A$ and contains a Cartan subalgebra $H$ with $H H \subseteq S$ then $S$ is a subalgebra of $A$ and is a Lie algebra under the multiplication in $A$. Therefore, in view of Theorem 1, we have

THEOREM 2. Let $A$ be a finite-dimensional flexible Lie-admissible algebra over a field of characteristic 0 . Let $R$ be the solvable radical of $A^{-}$and $S$ be a Levi-factor of $A^{-}$which is power-associative in A. Then $S$ is a Lie algebra under the multiplication in $A$ and is an ideal of $A$ if and only if $S$ contains a split Cartan subalgebra $H$ such that $H H \subseteq S$ and $[H, R]=0$. In this case, the multiplication in $A$ is given by (*).

Theorem 2 strengthens Theorem 4.1 in [2] which requires the additional assumptions that the radical $R$ of $A^{-}$is nilpotent in $A^{-}$and $H$ is nil in $A$. If, in Theorem 1, the complementary subalgebra $R$ of $A$ satisfies $[R, R]=R$, then the linear functionals $f_{i}$ are 0 . Thus we have

Corollary 1. Let $A, S, R$ be the same as in Theorem 1 and let $S$ be embedded as in Theorem 1. If $[R, R]=R$ then $R$ is an ideal of $A$. In particular, if $R^{-}$is a simple Lie algebra then $R$ is an ideal of $A$. 
COROllary 2. Let $A, S, R$ be the same as in Corollary 1 and of characteristic 0. If $R^{-}$is nilpotent and $R$ is nil in $A$ then $R$ is an ideal of $A$.

If $R^{-}$is nilpotent then ad $x$ is nilpotent in $A$ for all $x \in R$. Since $R$ is a nilalgebra, it follows from [2, Lemma 4.4] that the right multiplication $R(x)$ in $A$ by $x \in R$ is nilpotent. Hence the linear functionals $f_{i}$ are 0 and this proves Corollary 2.

\section{REFERENCES}

1. P. J. Laufer and M. L. Tomber, Some Lie admissible algebras, Canad. J. Math. 14 (1962), 287-292.

2. H. C. Myung, Some classes of flexible Lie-admissible algebras, Trans. Amer. Math. Soc. 167 (1972), 79-88.

3. A A subalgebra condition in Lie-admissible algebras, Proc. Amer. Math. Soc. $\$ 9$ (1976), 6-8.

4. G. B. Seligman, Modular Lie algebras, Ergebnisse der Math. und ihrer Grenzgebiete, Band 40, Springer-Verlag, New York, 1967.

5. R. M. Santilli, Lie-admissible approach to the hadronic structure, Volumes I, II, and III, Hadronic Press, Inc., Nonantum, Mass. (to appear).

Department of Mathematics, University of Northern Iowa, Cedar Falls, Iowa 50613 